\title{
Baseline low-density lipoprotein cholesterol to predict the extent of cardiovascular benefit from lipid-lowering therapies: A Review
}

\begin{abstract}
Eliano P Navarese ${ }^{1,2,3,4}$, MD, PhD; Felicita Andreotti ${ }^{5}, \mathrm{MD}, \mathrm{PhD}$; Paolo Raggi ${ }^{6} \mathrm{MD}, \mathrm{PhD}$; Michalina Kołodziejczak $^{2,7}$, MD; Antonino Buffon ${ }^{5}$, MD; PhD; Kevin Bliden ${ }^{1}$, MD; Udaya Tantry ${ }^{1}$, PhD; Jacek Kubica $^{7}, \mathrm{MD}, \mathrm{PhD}$; Gennaro Sardella ${ }^{8}$, MD; Alexander Lauten ${ }^{9}$, MD; Stefan Agewall ${ }^{10}$, MD, PhD, Paul A Gurbel $^{1}$, MD; Marc A. Brouwer ${ }^{11}$, MD.

${ }^{1}$ Interventional Cardiology and Cardiovascular Medicine Research, Inova Center for Thrombosis Research and Drug Development, Inova Heart and Vascular Institute, Falls Church, VA, USA; ${ }^{2}$ SIRIO MEDICINE network, evidence-based section, Falls Church, VA, USA; ${ }^{3}$ Interventional Cardiology and Cardiovascular Medicine Research, Cardiovascular Research Center, Mater Dei Hospital, Bari, Italy; ${ }^{4}$ Department of Medicine, University of Alberta, Edmonton, Canada; ${ }^{5}$ Institute of Cardiology, Catholic University Medical School, Rome, Italy; ${ }^{6}$ Mazankowski Alberta Heart Institute, University of Alberta, Edmonton, Alberta, Canada; ${ }^{7}$ Ludwik Rydygier Collegium Medicum, Nicolaus Copernicus University, Bydgoszcz, Poland; ${ }^{8}$ Department of Cardiovascular, Respiratory, Nephrology, Anesthesiology and Geriatric Sciences, "Sapienza" University of Rome, Rome, Italy; ${ }^{9}$ Department of Cardiology, Charité - Universitaetsmedizin Berlin, German Centre for Cardiovascular Research (DZHK)Department of Cardiology; ${ }^{10}$ Department of Cardiology, Oslo University Hospital and Institute of Clinical Medicine, Oslo University; ${ }^{11}$ Radboud University Medical Centre, Nijmegen, The Netherlands.
\end{abstract}

\section{Word count: 3348}

\section{Correspondence to:}

Eliano P. Navarese, MD, PhD

Interventional Cardiology and Cardiovascular Medicine Research

Inova Center for Thrombosis Research and Drug Development

Inova Heart and Vascular Institute

3300 Gallows Road

Falls Church, VA 22042

Email: elianonavarese@gmail.com or Eliano.Navarese@inova.org 


\begin{abstract}
\end{abstract}
Importance: Lipid-lowering therapies have been shown to improve cardiovascular outcome in a wide range of patients. The current guidelines recommend a graded approach to reduction in low- density lipoprotein cholesterol (LDL-C) proportional to the patient's risk, with the goal of achieving either a certain magnitude of reduction or a specific threshold of final LDL-C.
Observations: Recent findings from a meta-analysis of numerous randomized trials suggest that more attention should be given to the baseline LDL-C of an individual patient. In this review we discuss how the baseline LDL-C level may provide a means to better understand the results of recent cardiovascular outcome trials and the expected benefits of lipid-lowering therapies.
Conclusions and Relevance: The exact quantification of the clinical benefit associate with an intensified lipid-lowering therapy depends on the baseline LDL-C. Mortality is reduced in a $\log$ - linear fashion only when LDL-C >100 mg/dl. 


\section{Introduction}

Previous trials of statin therapy have shown that the benefits of treatment in terms of composite cardiovascular disease (CVD) event reduction are proportional to the magnitude of low-density lipoprotein cholesterol (LDL-C) lowering ${ }^{1}$. This evidence constitutes the foundation of current guidelines that support graded approach to LDL-C reductions. The European Society of Cardiology and European Atherosclerosis Society guidelines call for an LDL-C reduction to below $70 \mathrm{mg} / \mathrm{dL}-$ and/or at least a 50\% reduction — in patients at very high cardiovascular risk, while aiming for less than $100 \mathrm{mg} / \mathrm{dL}$ in patients at high risk $^{2}$. Guidelines from the American College of Cardiology and American Heart Association (ACC/AHA) do not have predefined goals ${ }^{3}$, but recommend appropriate intensity statins to reduce overall cardiovascular risk. During the last decade there has been an increased emphasis toward achieving ever lower LDL-C goals attained by intensifying statin therapy, and adding ezetimibe or more recently proprotein convertase subtilisin/kexin type 9 (PCSK9) inhibiting monoclonal antibodies ${ }^{2,3}$. This approach, however, while providing additional reductions in absolute composite cardiovascular endpoints, has resulted in diminishing returns in terms of reduction in mortality, that was not significantly reduced by more intensive treatment ${ }^{4}$. In contrast to the first lipid lowering trials, in the most recent ones, baseline LDL-C levels of the treated populations were lower ${ }^{5}$. Moreover, although the clinical benefits of lipid lowering per se are undisputed and supported by extensive randomized placebo controlled trials, there is heterogeneity in the magnitude of reductions in mortality and in cardiovascular outcomes among published trials.

\section{Heterogeneity between magnitude of cholesterol reduction and improved clinical outcomes}

Clinical benefit achieved with lipid lowering has been estimated based on a fixed linear association. The Cholesterol Treatment Trialists' (CTT) prospective meta-analysis of over 90,000 individual trial participants predicted a $21 \%$ relative reduction in the composite of coronary death, coronary revascularization, myocardial infarction, and stroke per $1 \mathrm{mmol} / \mathrm{l}(38.6 \mathrm{mg} / \mathrm{dL})$ reduction in LDL-C, 
with a significant $12 \%$ relative reduction in all-cause mortality and with enhanced benefit among

those with pre-existing coronary artery disease versus those without ${ }^{1,6}$. This prediction has largely influenced the design of clinical studies, including those testing the most recent therapeutic innovations such as the PCSK9 inhibitors. The recent Further Cardiovascular Outcomes Research With PCSK9 Inhibition in Subjects With Elevated Risk (FOURIER) trial ${ }^{7}$ randomly assigned 27,564 patients with cardiovascular disease, already on a moderate- to high-intensity statin regimen, to receive, 1:1, either subcutaneous injections of evolocumab or matching placebo. FOURIER lasted a median of 2.2 years (maximum 3 years). Evolocumab reduced LDL-C from 92 to $30 \mathrm{mg} / \mathrm{dL}$ and was accompanied by a $15 \%$ relative reduction of the primary composite endpoint of non-fatal myocardial infarction, stroke, hospitalization for angina, revascularization or cardiovascular death (hazard ratio (HR), 95\% confidence interval (CI): 0.85, 0.79-0.92). When measured per $40 \mathrm{mg} / \mathrm{dL}$ $(\sim 1 \mathrm{mmol} / \mathrm{l})$ reduction in LDL-C, treatment with evolocumab reduced the hazard of the primary endpoint by $11.0 \%$ (HR $0.89,95 \%$ CI $0.84-0.94$ ); thus, the magnitude of this effect was less than the expected $21 \%$ risk reduction predicted by CTT, with no significant effect on cardiovascular mortality. In the recently released ODYSSEY Outcomes trial (Alirocumab in Patients After Acute Coronary Syndrome) patients with recent acute coronary syndrome were randomized to another PCSK9 inhibitor, alirocumab, or placebo on top of maximum tolerated doses of statins ${ }^{8,9}$. After a median follow-up of 2.8 years, LDL-C levels were $53.3 \mathrm{mg} / \mathrm{dL}$ in the alirocumab group compared to $101.4 \mathrm{mg} / \mathrm{dL}$ in the placebo group. The primary endpoint - time to coronary death, myocardial infarction, hospitalization for unstable angina or ischemic stroke - was $15 \%$ lower with alirocumab versus placebo (HR $0.85,95 \%$ CI $0.78-0.93 \%$ ). In a hierarchical analysis, total mortality was reduced by $15 \%$ (nominal p-value 0.026 ) while $\mathrm{CV}$ and coronary artery disease (CHD) death were not significantly reduced. Importantly, a reduction in total mortality was noted only in the subgroup of patients with baseline LDL-C $>100 \mathrm{mg} / \mathrm{dl}$. At variance with the above studies that enrolled high risk patients, the Justification for the Use of Statins in Prevention: an Intervention Trial Evaluating 
Rosuvastatin (JUPITER) trial, conducted in primary prevention ${ }^{10}$, did demonstrate a total mortality benefit at 2 years with a $55 \mathrm{mg} / \mathrm{dL}$ reduction in LDL-C obtained with rosuvastatin, similar in magnitude to the LDL-C reduction observed in FOURIER at 2.2 years. In aggregate, these results lend support to the interpretation that factors other than the magnitude of LDL-C reduction or the patients' clinical risk are at play and may deserve consideration in order to effectively quantify the expected clinical benefit from lipid-lowering therapy.

\section{Trial length}

It has been argued that the attenuated observed versus expected overall benefit and the lack of mortality benefit with evolocumab versus placebo may be due to the relatively short trial duration. Duration, however, does not appear to be a sufficient explanation based on examination of previous statin trials, for instance the Incremental Decrease in End Points Through Aggressive Lipid Lowering Study Group (IDEAL) ${ }^{4}$, which lasted approximatively 5 years and failed to demonstrate an all-cause or cardiovascular death reduction benefit, despite an LDL-C lowering of $41 \mathrm{mg} / \mathrm{dl}$. The argument is also not supported by the IMProved Reduction of Outcomes: Vytorin Efficacy International Trial (IMPROVE IT) ${ }^{11}$, which had longer follow-up (7 years) and showed a modest $7 \%$ relative reduction $(\mathrm{HR}=0.93$, 95\% CI $0.89-0.99)$ in the primary composite endpoint of cardiovascular death, myocardial infarction, unstable angina requiring hospitalization, coronary revascularization, or stroke when ezetimibe was added to simvastatin. IMPROVE-IT again did not show a survival benefit (HR 0.99, 95\% CI 0.91-1.07). Thus, trial duration does not appear to explain the lack of mortality benefit observed in FOURIER. In both trials, relative risk reductions were more pronounced at longer time frames, with greater relative risk reduction observed in year 2 compared to year 1 of follow-up. However, although a longer follow-up can have contributed to an increased effect observed over time, it is unlikely that the magnitude of reduction would outstrip 
that predicted by the CTT meta-analysis, which remains consistent for each year, being the same at year 5 as the magnitude observed in years 1 and 2.

\section{Baseline LDL-C levels across lipid-lowering strategies}

The heterogeneous benefit on mortality and morbidity noted in various trials could be ascribed in part to differences in pre-treatment LDL-C levels of the randomized populations. In fact, the baseline LDL-C levels were considerably different across lipid-lowering strategies tested over the last 10-15 years. For instance, the JUPITER ${ }^{10}$ enrolled subjects for primary prevention with a median baseline LDL-C of $108 \mathrm{mg} / \mathrm{dL}$; the study demonstrated a total mortality benefit at 2 years with a $55 \mathrm{mg} / \mathrm{dL}$ reduction in LDL-C. In the CTT meta-analysis the average baseline LDL-C was $120 \mathrm{mg} / \mathrm{dL}$. In contrast, in the IMPROVE IT trial $(93.8 \mathrm{mg} / \mathrm{dL})$, the FOURIER trial $(92 \mathrm{mg} / \mathrm{dL})$ and the ODYSSEY Outcomes trial $(87 \mathrm{mg} / \mathrm{dL})$, the mean baseline LDL-C levels in the more intensive lipid-lowering arms were lower than in JUPITER or in CTT (146.6 mg/dL) and fairly similar across trials; despite LDL-C reductions ranging from 40.6 to $62 \mathrm{mg} / \mathrm{dl}$, none of them showed significant reductions in cardiovascular mortality with more intensive lipid- lowering.

The Stroke Prevention by Aggressive Reduction in Cholesterol Levels (SPIRE)-1 was shorter (median 7 months) and had lower entry LDL-C levels for eligibility (mean $94 \mathrm{mg} / \mathrm{dL}$ ) than SPIRE-2 (baseline LDL-C >100 mg/dL, mean $134 \mathrm{mg} / \mathrm{dL}$, median 1 year duration) ${ }^{12}$. In SPIRE-2, the primary composite endpoint (including cardiovascular death) was the same as FOURIER's, and was reduced at 1 year by $21 \%$ (HR $0.79,95 \%$ CI $0.65-0.97, \mathrm{p}=0.02$ ). This was close to what would be expected, based on a mean LDL-C reduction of $62 \mathrm{mg} / \mathrm{dL}(1.6 \mathrm{mmol} / \mathrm{L})$, according to the CTT prediction algorithm (21\% relative reduction in the composite of coronary death, coronary revascularization, myocardial infarction, and stroke per $1 \mathrm{mmol} / \mathrm{l})$. These observations were the impetus for an in-depth analysis of the evidence used to support current guidelines on lipid management ${ }^{13}$. 
A recently published in-depth analysis provides a compelling and comprehensive demonstration of the role of baseline LDL-C in influencing clinical outcomes among patients treated with LDL-Clowering agents ${ }^{13}$ in primary and secondary prevention trials. The analysis offers the opportunity to reconcile the apparent inconsistencies among the available trials concerning current LDL-C metrics and clinical outcomes. The findings support individualizing the estimates of potential cardiovascular risk reduction derived from LDL-C lowering therapy based not only on a patient's clinical risk and magnitude of LDL-C reduction, but also on the patient's baseline LDL-C level and on the outcome of interest. The analysis clearly demonstrated an association between LDL-C lowering and all-cause mortality benefit as a function of baseline LDL-C. For each $40 \mathrm{mg} / \mathrm{dL}$ higher baseline LDL-C, more intensive LDL-C reduction was associated with an additional 9\% reduction in all-cause mortality (rate ratio (RR) $0.91,95 \%$ CI $0.86-0.96, p=0.001$ ) but only when baseline LDL-C levels were $>100 \mathrm{mg} / \mathrm{dL}$. Similarly, an association emerged between LDL-C lowering and cardiovascular mortality benefit as a function of baseline LDL-C. For each $40 \mathrm{mg} / \mathrm{dL}$ higher baseline LDL-C, more intensive LDL-C lowering therapy was associated with an additional 14\% reduction in cardiovascular mortality (RR 0.86, 95\% CI 0.80-0.94, p<0.0001) but only when baseline LDL-C levels were $>100 \mathrm{mg} / \mathrm{dL}^{13}$. A reduction in the risk of myocardial infarction, revascularization and major cardiovascular events was also greater with increasing baseline LDL-C level, whereas the risk reduction in cerebrovascular events and ischemic stroke was similar across baseline LDL-C level. Thus, the most consistent and compelling finding of this analysis was that baseline LDL-C emerged as a key driver of the magnitude of relative benefit derived from LDL-C lipid lowering therapies with regard to reduction in all-cause and cardiovascular mortality. In fact, LDL-C at baseline explained $61 \%$ and $60 \%$ of the observed between-trial-variance, respectively. The observed treatment effect in relation to baseline LDL-C was robust across multiple sensitivity analyses. 
The graded mortality benefits observed with more versus less intensive lipid-lowering therapy for different pretreatment LDL-C levels translate into different numbers needed to treat (NNTs) across the explored subgroups (Figures 1A and 1B). Building on this observation, the use of more intensive lipid-lowering strategies can lead to the most meaningful reduction of all-cause and cardiovascular mortality when baseline LDL-C is equal to or higher than $100 \mathrm{mg} / \mathrm{dL}$, with NNTs as low as 53 and 57, respectively (Figures 1A and 1B, baseline LDL-C subgroup 160 $\mathrm{mg} / \mathrm{dL}$ ). In contrast, very high NNTs are observed for all-cause and cardiovascular mortality reduction when the baseline LDL-C is less than $100 \mathrm{mg} / \mathrm{dL}$ (Figures $1 \mathrm{~A}$ and 1B, baseline LDL-C subgroup $<100 \mathrm{mg} / \mathrm{dL}$ ).

The effectiveness of lipid lowering therapy in reducing events has often been interpreted as a function of LDL-C reduction induced by different therapeutic agents rather than a function of baseline LDL- $\mathrm{C}^{14}$. The above-mentioned recent analysis showed in multivariate meta-regressions that baseline LDL-C is associated with mortality and cardiovascular event reduction independently of the magnitude of LDL-C reduction, and largely determines the association with total and cardiovascular mortality reduction ${ }^{13}$. In short, for a given magnitude of LDL-C reduction, the decline in cardiovascular and all-cause mortality achieved with lipid lowering agents are greater with higher baseline LDL-C levels (Figure 2 and suppl Figure 1). Based on this relation, it is possible to imagine a clinical scenario where two patients being treated with a lipid-lowering drug can reach the same proportional LDL-C reduction, but attain different results due to their pretreatment LDL-C levels. In this context, patient A with a lower baseline LDL-C (baseline LDL-C= $98 \mathrm{mg} / \mathrm{dL}, 50 \%$ LDL-C reduction= $49 \mathrm{mg} / \mathrm{dL}$ ) will derive a markedly lower cardiovascular mortality benefit with a more intensive lipid-lowering strategy compared to patient B with higher baseline LDL-C (baseline LDL-C=150 $\mathrm{mg} / \mathrm{dL}$ ): $10.36 \%$ vs $22.51 \%$ proportional risk reductions, respectively (Figure 2). 
This may contribute to explain the lack of mortality benefit in the more recent trials of intensive lipid lowering therapies. Importantly, a greater risk reduction in myocardial infarction, revascularization and composite cardiovascular events occurred with higher baseline LDL-C levels. In support of this interpretation of the data the post-hoc analysis of the ODYSSEY Outcomes suggested a greater influence of alirocumab versus placebo on all-cause death (HR $0.71,95 \%$ CI 0.56-0.90), cardiovascular mortality (HR 0.69, 95\% CI 0.52-0.92), and overall major cardiovascular events (HR 0.76, 95\% CI 0.65-0.87) in patients with baseline LDL-C of $100 \mathrm{mg} / \mathrm{dL}$ or greater compared to those with baseline LDL-C below $100 \mathrm{mg} / \mathrm{dL}$, although the interaction term was nonsignificant for all-cause death ${ }^{15}$.

\section{Baseline LDL-C and mortality benefit with PCSK9 inhibitors}

PCSK9 inhibitors are an innovative lipid-lowering class. The first evidence of the effectiveness of PCSK9 antibodies was observed in a meta-analysis of 24 phase II and III trials involving a total of 10,159 patients that showed a significant reduction in all-cause mortality in patients treated with a PCSK9 inhibitor ${ }^{16}$. Of note, no signal for heterogeneity was present across trials in the analysis of all-cause and cardiovascular mortality, and there was stability of the direction of results in the sensitivity analyses. The mean baseline LDL-C level was $142.9 \mathrm{mg} / \mathrm{dL}$. The finding of reduced mortality was not observed in the more recent outcome-based studies with PCSK9 antibodies, such as FOURIER that tested evolocumab versus placebo. There was a reduction in all-cause mortality but not in cardiovascular mortality in the more recent ODYSSEY Outcomes trial in which patients received alirocumab or placebo. In both trials patients were on optimized statin therapy; in the ODYSSEY Outcomes, to maximize the number of patients in the target range, alirocumab was uptitrated to $150 \mathrm{mg}$ every two weeks in patients with LDL-C $\geq 50 \mathrm{mg} / \mathrm{dL}$. In the FOURIER study, average baseline LDL-C was $92 \mathrm{mg} / \mathrm{dL}$ while in the ODYSSEY Outcomes it was $87 \mathrm{mg} / \mathrm{dL}$. In the ODYSSEY Outcomes trial subgroup analysis, patients presenting with baseline LDL-C $\geq 100$ 
$\mathrm{mg} / \mathrm{dL}$ showed a more pronounced benefit in cardiovascular and overall mortality. A recent metaanalysis $^{16}$ and the ODYSSEY Outcomes subgroup analysis are in agreement that the mortality benefit with PCSK9 inhibitors is related to pre-treatment LDL-C levels.

\section{Mortality with PCSK9 inhibitors by baseline LDL-C}

In an updated analysis in this review of a previous report on PCSK9 inhibitors ${ }^{16}$, the addition of the most recent studies stratified by baseline LDL-C confirm the significant cardiovascular and allcause mortality benefit with PCSK9 inhibitors in subjects with LDL-C equal or greater than 100 $\mathrm{mg} / \mathrm{dL}$ (Figure 3 and suppl Figure 2).

\section{Integrated prediction model to quantify the net clinical benefit}

The new evidence discussed so far may have important implications for daily practice and future research. First, a new model to predict absolute atherosclerotic cardiovascular disease risk reduction will have to be introduced to take into account baseline LDL-C in addition to LDL-C reduction. The expected absolute benefit, expressed as the NNT, will depend not only on the a-priori absolute clinical risk and magnitude of LDL-C reduction, but also on the baseline LDL-C level and the corresponding estimated relative risk reduction in a specific event or composite outcome (Figures 1A-1C).

Second, these new insights provide information into the cost-effectiveness of (expensive) non-statin drugs, and may help identify patients for whom PCSK9 inhibitors may be considered high-value care.

Third, the design of future trials requires important modifications, and should incorporate the possibility to gain further insight into the association between baseline LDL-C and outcomes. A methodological prerequisite is to adjust for other confounders. 
The recent metanalysis ${ }^{13}$ supports the use of a patient's baseline LDL-C level to estimate the potential risk reduction and to decide when to add a non-statin therapy. Focusing on the CTT conclusions (a relative risk reduction for a composite outcome for each 1 mmol decrease in LDL-C) may obscure the $14 \%$ additional reduction in the risk of cardiovascular death per $40 \mathrm{mg} / \mathrm{dL}$ increase in baseline LDL-C. In the CTT meta-analysis, the majority of patients were enrolled in trials conducted before 2010, with high baseline LDL-C; this is also reflected by the wide confidence intervals observed in the CTT's lowest baseline LDL-C level group ( $<2 \mathrm{mmol} / \mathrm{L})$. The CTT analysis had considerably fewer patients in the lowest baseline LDL-C group compared to the other groups.

Finally, the recent metanalytic findings may have an impact on public health and an effect on cost-effectiveness evaluation. An updated Institute for Clinical and Economic Review (ICER) revision of PCSK9 monoclonal antibodies is currently warranted to provide the most precise costefficacy ratio associated with PCSK9 inhibitors. In light of the above results, the current ICER recommendations may be underestimating the potential benefit of PCSK9 monoclonal antibodies for patients with elevated baseline LDL-C levels. The preliminary findings of a mortality benefit among patients with baseline LDL-C $\geq 100 \mathrm{mg} / \mathrm{dL}$ corroborate the quantitative interaction principle $^{17}$, whereby the benefit of treatment is larger among high-risk patients, where risk can be interpreted both as clinical condition (eg concomitant cardiovascular disease or familiar hypercholesterolemia) as well as a biological risk due to high baseline LDL-C. As we enter the era of personalized medicine, treatment decisions can, and should, be based on an individual patient's clinical risk, baseline LDL-C level, LDL-C reduction, and the anticipated benefits from added therapy.

\section{Vulnerable LDL-C threshold effect}


Since the baseline LDL-C cholesterol level has been described to influence mortality independently

of the magnitude of LDL-C reduction ${ }^{13}$ but only when LDL-C is $\geq 100 \mathrm{mg} / \mathrm{dL}$, it is plausible to consider this threshold as a marker of instability. There is a close link between LDL-C levels, plaque progression, and plaque instability ${ }^{18}$ (Figure 4). Serial intravascular ultrasound (IVUS) data analysis indicated that statin therapy was less effective in inducing coronary plaque regression in patients with low cholesterol levels but more effective in those with high cholesterol levels at baseline $^{19}$. Plaque stabilizing characteristics are also less likely in those with higher LDL-C levels ${ }^{20}$.

The above observations emphasize consideration on the possible underlying pathogenetic mechanisms related to the pretreatment amount of LDL-C that contribute to this effect. When high levels of cholesterol occur in the bloodstream, excess LDL-C begins to enter the inner wall of the artery. Eventually the deposited cholesterol hardens into a plaque prone to rupture or erosion, which are substrates for coronary thrombosis and ST elevation myocardial infarction ${ }^{21}$. Plaque destabilization can be therefore the direct consequence of increased amount of LDL-C before treatment which can in turn lead to higher inflammatory reactions and a host of destabilizing mechanisms: the cholesterol uptake from apolipoprotein B containing lipoproteins such as LDL-C and oxidation of native LDL-C by oxygen-free radicals stimulates the expression of several inflammatory markers and induces the expression of molecules on endothelial cells that promote adhesion of monocytes . This cascade is also triggered by an increase of the PCSK9 enzyme during an acute coronary syndrome ${ }^{22}$. The involvement of cholesterol crystals in plaque progression and destabilization of atherosclerotic plaques has also been recently recognized as another harbinger of plaque instability; they can perforate the intimal surface overlying ruptured plaques ${ }^{23}$. The above mentioned effects are proven related to the amount of LDL-C exposure ${ }^{23}$. Thus, a heightened inflammatory status might enhance the plaque destabilization leading to increased adverse events and mortality, particularly during an acute coronary syndrome episode. There is initial evidence that 
the increased LDL-C above $100 \mathrm{mg} / \mathrm{dl}$ is related to inflammatory biomarkers ${ }^{24}$. This might further corroborate the role of a possible pro-inflammatory threshold effect sustained by the pretreatment LDL-C of and above $100 \mathrm{mg} / \mathrm{dL}$. The exact interplay between LDL-C and inflammation in causing plaque destabilization is still to be elucidated; to show the changes in the relation between the inflammatory data response and the baseline LDL-C below vs equal or above $100 \mathrm{mg} / \mathrm{dl}$ with modern intensified lipid-lowering agents would be vital to more deeply characterize the relative influence of intensified therapies on these pathways and progression/destabilization of coronary plaque.

\section{Conclusions}

The exact quantification of the clinical benefit associate with an intensified lipid-lowering therapy depends on the baseline LDL-C that reduces mortality in a log-linear fashion only when LDL-C $\geq 100 \mathrm{mg} / \mathrm{dl}$. This observation should prompt future investigations to elucidate possible vulnerable threshold effect of baseline $100 \mathrm{mg} / \mathrm{dL}$ LDL-C that might trigger of coronary plaque destabilization.

\section{Perspectives}

Future lipid-lowering studies should help generate new models of personalized medicine which ultimately integrate patient baseline LDL-C , magnitude of LDL-C reduction and risk profile. These models should target LDL-C with the potential to usher in a new era of individualized treatment strategies that maximize the clinical efficacy of a lipid-lowering strategy while minimizing the associated costs. 


\section{References}

1. Baigent C, Blackwell L, Emberson J, et al. Efficacy and safety of more intensive lowering of LDL cholesterol: a meta-analysis of data from 170,000 participants in 26 randomised trials. Lancet (London, England). Nov 13 2010;376(9753):1670-1681.

2. Catapano AL, Graham I, De Backer G, et al. 2016 ESC/EAS Guidelines for the Management of Dyslipidaemias: The Task Force for the Management of Dyslipidaemias of the European Society of Cardiology (ESC) and European Atherosclerosis Society (EAS) Developed with the special contribution of the European Assocciation for Cardiovascular Prevention \& Rehabilitation (EACPR). Atherosclerosis. Oct 2016;253:281-344.

3. Stone NJ, Robinson JG, Lichtenstein AH, et al. 2013 ACC/AHA guideline on the treatment of blood cholesterol to reduce atherosclerotic cardiovascular risk in adults: a report of the American College of Cardiology/American Heart Association Task Force on Practice Guidelines. Journal of the American College of Cardiology. Jul 1 2014;63(25 Pt B):2889-2934.

4. Pedersen TR, Faergeman O, Kastelein JJ, et al. High-dose atorvastatin vs usual-dose simvastatin for secondary prevention after myocardial infarction: the IDEAL study: a randomized controlled trial. Jama. Nov 16 2005;294(19):2437-2445.

5. Kazi DS, Penko J, Coxson PG, et al. Updated Cost-effectiveness Analysis of PCSK9 Inhibitors Based on the Results of the FOURIER Trial. Jama. Aug 22 2017;318(8):748-750.

6. Baigent C, Keech A, Kearney PM, et al. Efficacy and safety of cholesterol-lowering treatment: prospective meta-analysis of data from 90,056 participants in 14 randomised trials of statins. Lancet (London, England). Oct 8 2005;366(9493):1267-1278.

7. Sabatine MS, Giugliano RP, Keech AC, et al. Evolocumab and Clinical Outcomes in Patients with Cardiovascular Disease. The New England journal of medicine. May 4 2017;376(18):1713-1722.

8. Schwartz GG, Bessac L, Berdan LG, et al. Effect of alirocumab, a monoclonal antibody to PCSK9, on long-term cardiovascular outcomes following acute coronary syndromes: rationale and design of the ODYSSEY outcomes trial. American heart journal. Nov 2014;168(5):682-689.

9. Comparison of transcatheter versus surgical aortic valve replacement in younger low surgical risk patients with severe aortic stenosis (NOTION-2). NCT02825134. .

10. Ridker PM, Danielson E, Fonseca FA, et al. Rosuvastatin to prevent vascular events in men and women with elevated C-reactive protein. The New England journal of medicine. Nov 20 2008;359(21):2195-2207.

11. Cannon CP, Blazing MA, Giugliano RP, et al. Ezetimibe Added to Statin Therapy after Acute Coronary Syndromes. The New England journal of medicine. Jun 18 2015;372(25):2387-2397.

12. Ridker PM, Revkin J, Amarenco P, et al. Cardiovascular Efficacy and Safety of Bococizumab in HighRisk Patients. The New England journal of medicine. Apr 20 2017;376(16):1527-1539.

13. Navarese EP RJ, Kowalewski M, Kołodziejczak M, Andreotti F, Bliden K, Tantry U, Kubica J, Raggi P, Gurbel PA. Association Between Baseline LDL-C Level and Total and Cardiovascular Mortality After LDL-C Lowering A Systematic Review and Meta-analysis. Jama. 2018;319(15):1566-1579.

14. Silverman MG, Ference BA, Im K, et al. Association Between Lowering LDL-C and Cardiovascular Risk Reduction Among Different Therapeutic Interventions: A Systematic Review and Meta-analysis. Jama. Sep 27 2016;316(12):1289-1297.

15. Steg PG. The ODYSSEY OUTCOMES Trial: Topline Results Alirocumab in Patients After Acute Coronary Syndrome. American College of Cardiology - 67th Scientific Sessions March 10, 2018. 
16. Navarese EP, Kolodziejczak M, Schulze V, et al. Effects of Proprotein Convertase Subtilisin/Kexin

17. Califf RM. Issues facing clinical trials of the future. Journal of internal medicine. Nov 2003;254(5):426-433.

18. Libby P, Pasterkamp G. Requiem for the 'vulnerable plaque'. European heart journal. Nov 14 2015;36(43):2984-2987.

19. Wakabayashi K, Nozue T, Yamamoto S, et al. Efficacy of Statin Therapy in Inducing Coronary Plaque Regression in Patients with Low Baseline Cholesterol Levels. Journal of atherosclerosis and thrombosis. Sep 1 2016;23(9):1055-1066.

20. Kataoka $\mathrm{Y}$, Hammadah $\mathrm{M}$, Puri R, et al. Plaque microstructures in patients with coronary artery disease who achieved very low low-density lipoprotein cholesterol levels. Atherosclerosis. Oct 2015;242(2):490-495.

21. Virmani R, Burke AP, Farb A. Plaque rupture and plaque erosion. Thrombosis and haemostasis. Sep 1999;82 Suppl 1:1-3.

22. Navarese EP, Kolodziejczak M, Kereiakes DJ, Tantry US, O'Connor C, Gurbel PA. Proprotein Convertase Subtilisin/Kexin Type 9 Monoclonal Antibodies for Acute Coronary Syndrome: A Narrative Review. Annals of internal medicine. May 3 2016;164(9):600-607.

23. Dai J, Tian J, Hou J, et al. Association between cholesterol crystals and culprit lesion vulnerability in patients with acute coronary syndrome: An optical coherence tomography study. Atherosclerosis. Apr 2016;247:111-117.

24. Lakkur S, Judd S, Bostick RM, et al. Oxidative stress, inflammation, and markers of cardiovascular health. Atherosclerosis. Nov 2015;243(1):38-43. 


\section{LEGEND}

Figure 1A. Absolute risk reduction (ARR) and number needed to treat (NNT) analysis of all-cause mortality stratified by baseline LDL-C. ARR is negative and NNT not calculable for the first pair of bars.

Figure 1B. Absolute risk reduction (ARR) and number needed to treat (NNT) analysis of cardiovascular mortality stratified by baseline LDL-C.

Figure 1C. Absolute risk reduction (ARR) and number needed to treat (NNT) analysis of major adverse cardiovascular events (MACE) stratified by baseline LDL-C. LDL=low density lipoprotein.

Figure 2. Meta-regression analysis of proportional risk reduction of cardiovascular mortality by baseline LDL-C. Case A and B represent hypothetical patient cases who experience the same magnitude of LDL-C reduction but with different baseline LDL-C levels.

Risk reduction has been estimated according to a multivariate equation that consider s baseline LDL-C and magnitude of LDL-C reduction (ref 13). LDL=low density lipoprotein.

Figure 3. Updated meta-analysis of cardiovascular mortality across PCSK9 inhibitor studies by baseline LDL-C. MH=Mantel-Henzel. LDL=low density lipoprotein.

Figure 4. Association between atherosclerotic plaque composition and baseline LDL-C level and intensification of lipid-lowering therapy. A) Pre-treatment LDL-C $<100 \mathrm{mg} / \mathrm{dL}$ and stable plaque, B) Pre-treatment LDL-C $\geq 100 \mathrm{mg} / \mathrm{dL}$ and vulnerable plaque 


\section{Figure 1A}

1

2

3

4

5

6

7

8

9

10

11

12

13

14

15

16

17

18

19

20

21

22

23

24

25

26

27

28

29

30

31

32

33

34

35

36

37

38

39

40

41

42

43

44

45

46

47

48

49

50

51

52

53

54

55

56

57

58

59

60

61

62

63

64

65

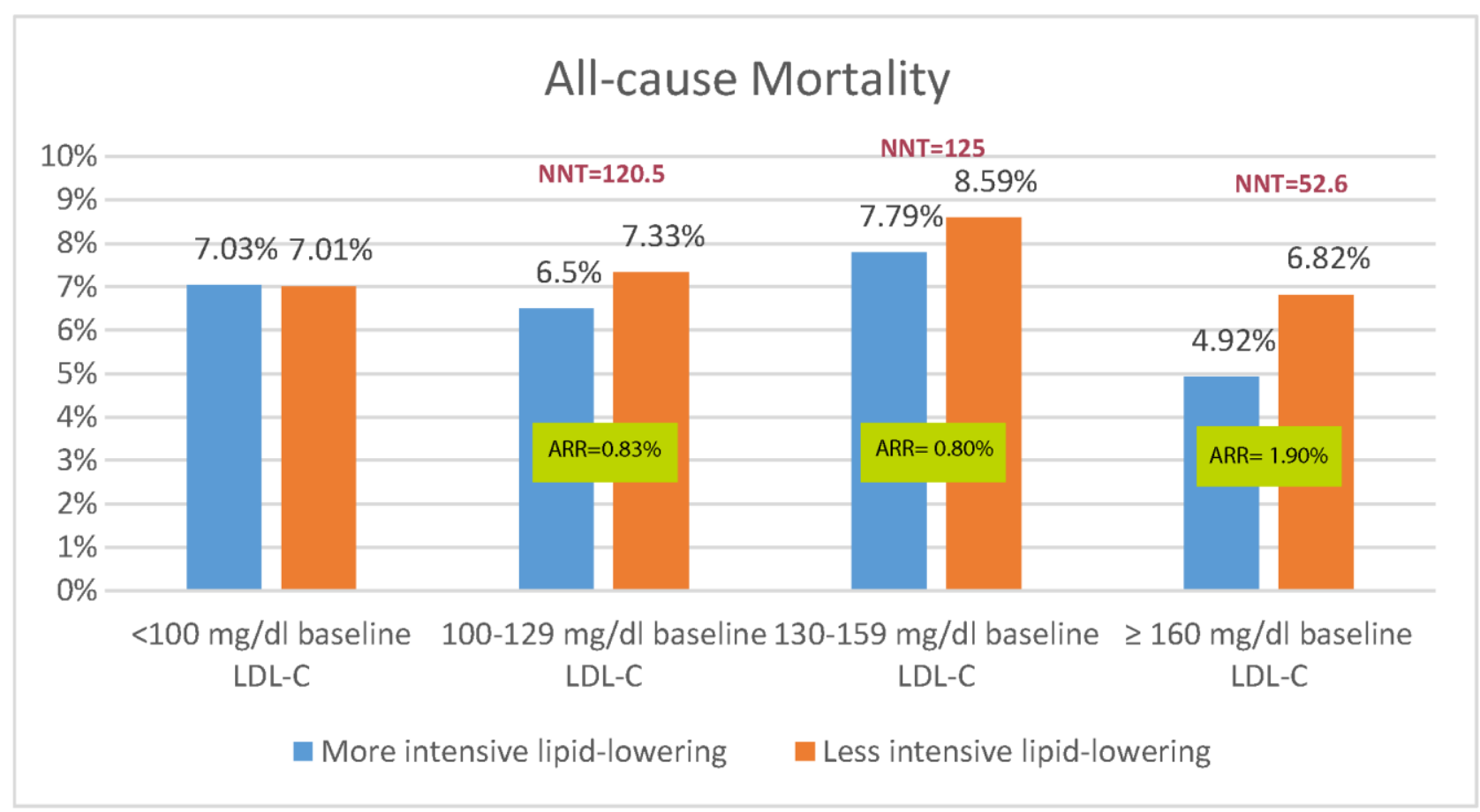




\section{Figure 1B}

1

2

3

4

5

6

7

8

9

10

11

12

13

14

15

16

17

18

19

20

21

22

23

24

25

26

27

28

29

30

31

32

33

34

35

36

37

38

39

40

41

42

43

44

45

46

47

48

49

50

51

52

53

54

55

56

57

58

59

60

61

62

63

64

65

\section{Mortality}

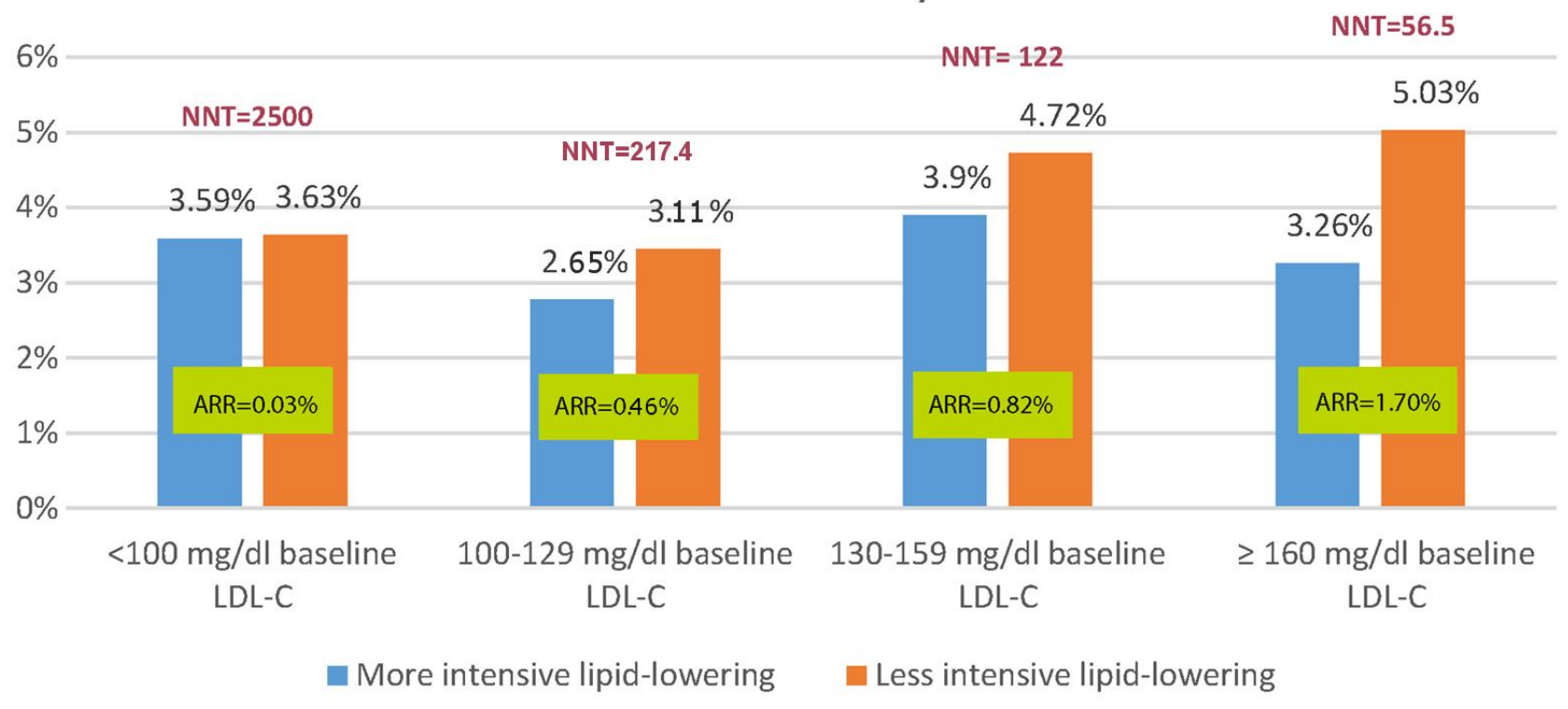




\section{Figure 1C}

1

2

3

4

5

6

7

8

9

10

11

12

13

14

15

16

17

18

19

20

21

22

23

24

25

26

27

28

29

30

31

32

33

34

35

36

37

38

39

40

41

42

43

44

45

46

47

48

49

50

51

52

53

54

55

56

57

58

59

60

61

62

63

64

65

\section{MACES}

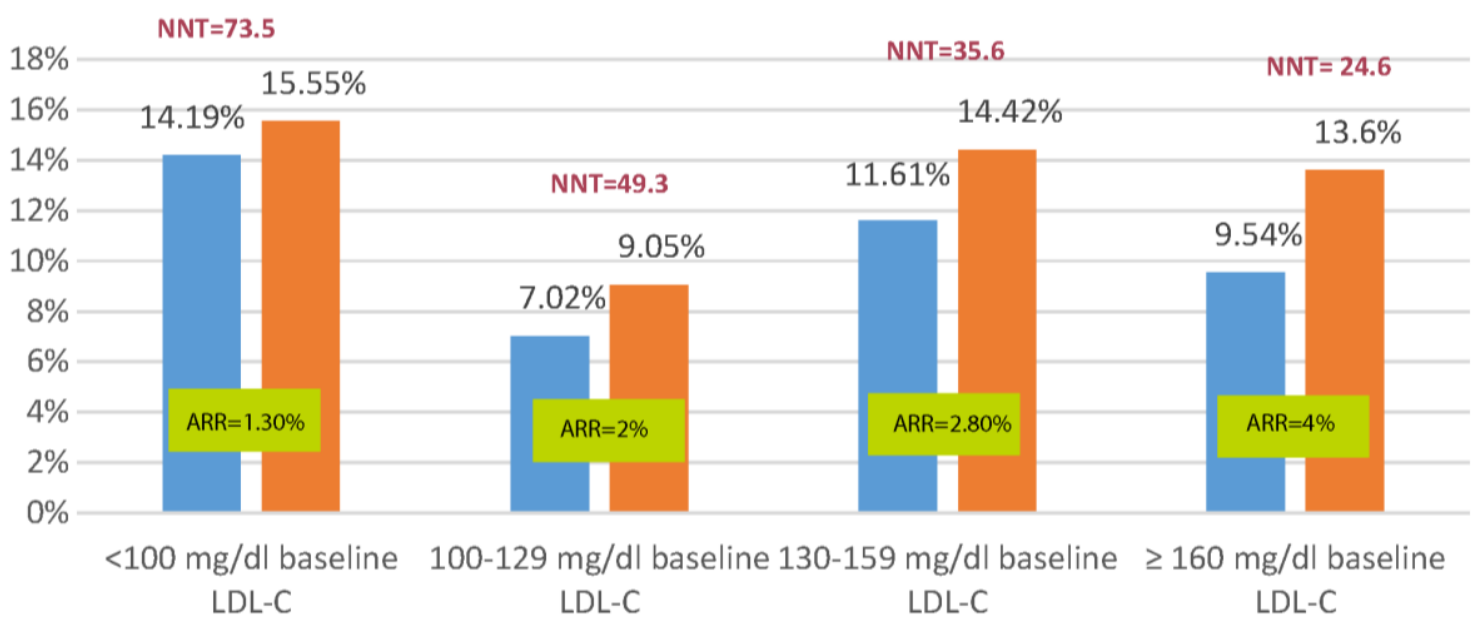

a More intensive lipid-lowering $\quad$ Less intensive lipid-lowering 
Figure 2

1

2

3

4

5

6

7

8

10

11

12

13

14

15

16

17

18

19

20

21

22

23

24

25

26

27

28

29

30

31

32

33

34

35

36

37

38

39

40

41

42

43

44

45

46

47

48

49

50

51

52

53

54

55

56

57

58

59

60

61

62

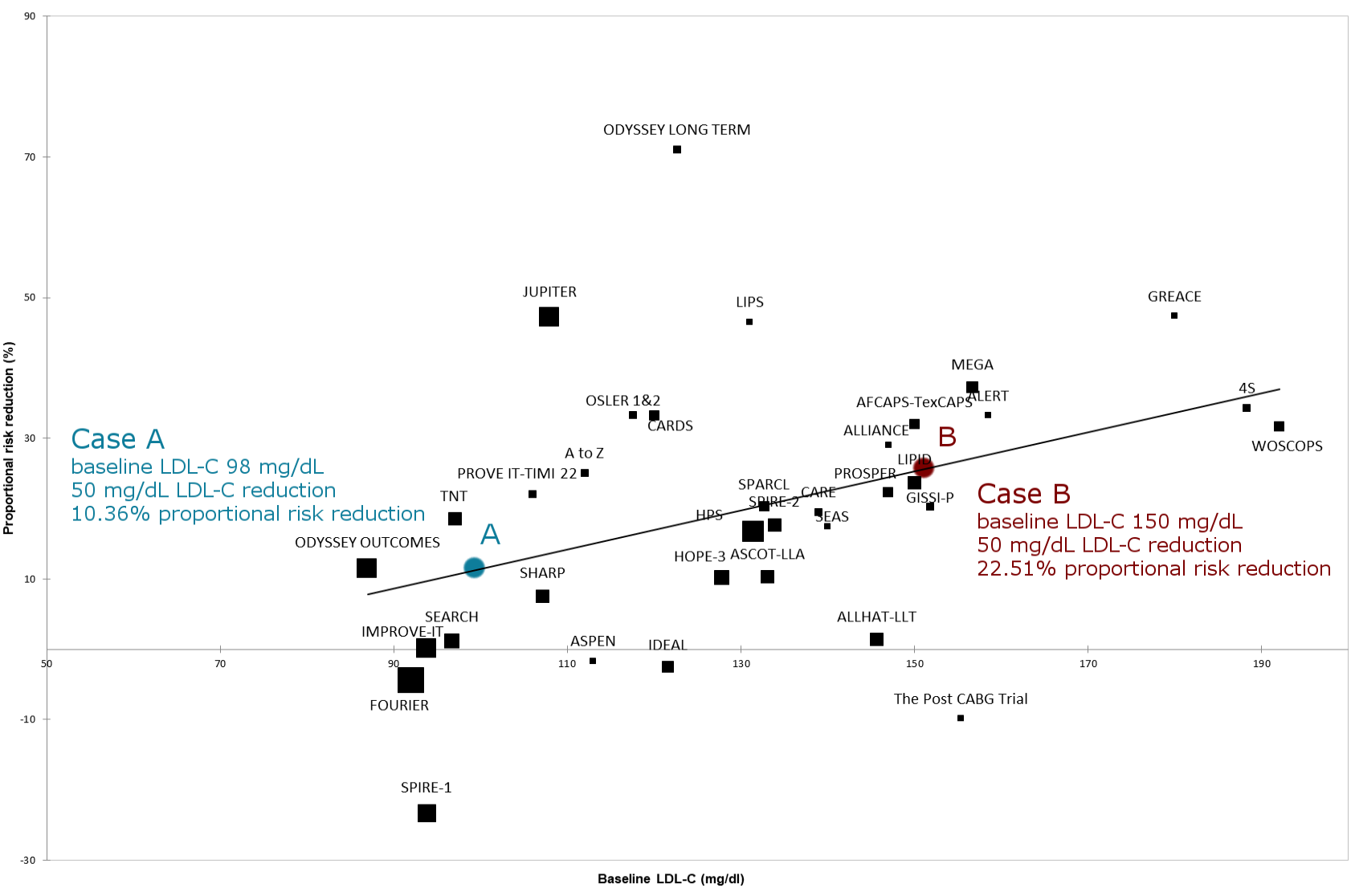




\section{Figure 3}




\section{Figure 4}

1

2

3

4

5

6

7

8

9

10

11

12

13

14

15

16

17

18

19

20

21

22

23

24

25

26

27

28

29

30

31

32

33

34

35

36

37

38

39

40

41

42

43

44

45

46

47

48

49

50

51

52

53

54

55

56

57

58

59

60

61

62

63

64

65
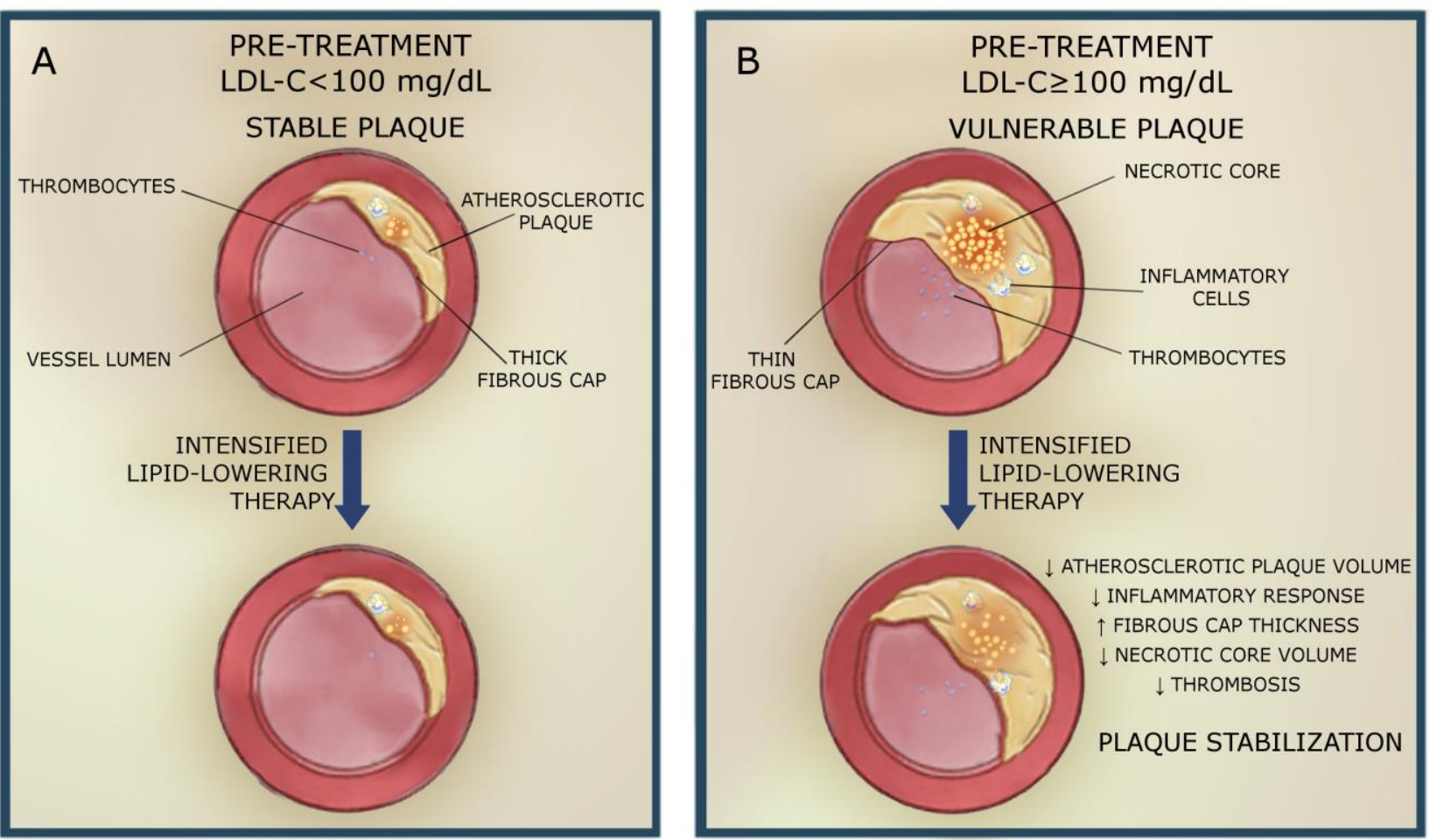


\section{Mortality}

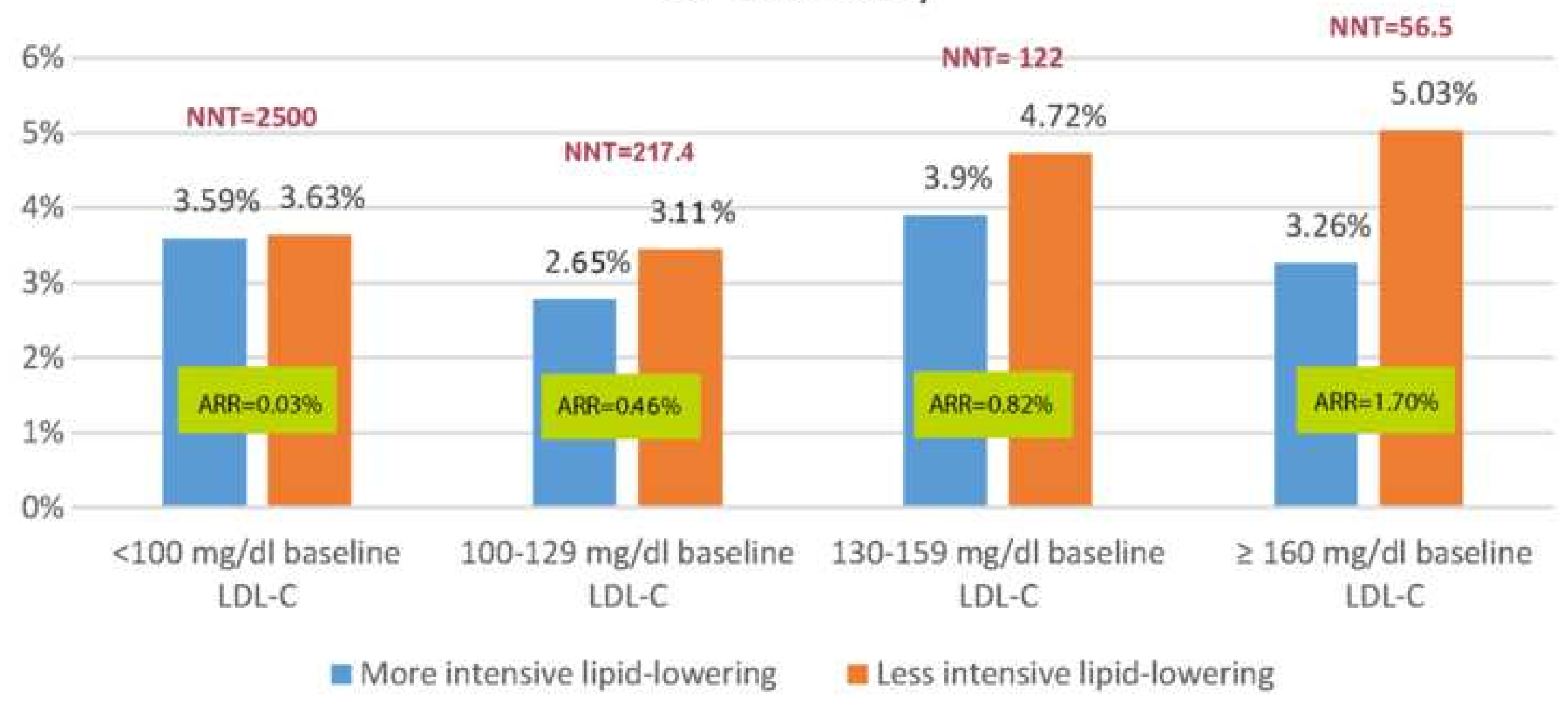



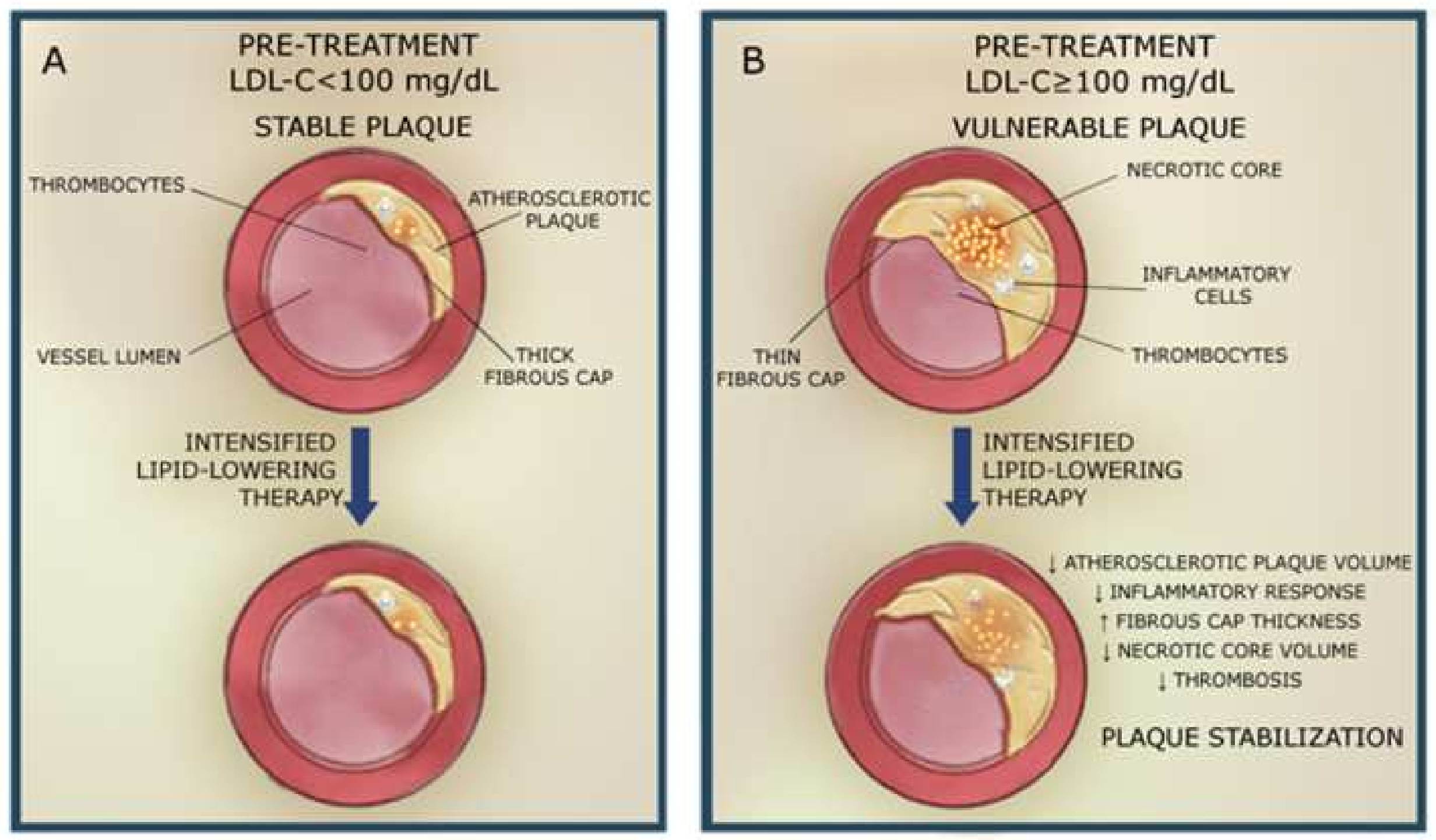


\section{All-cause Mortality}

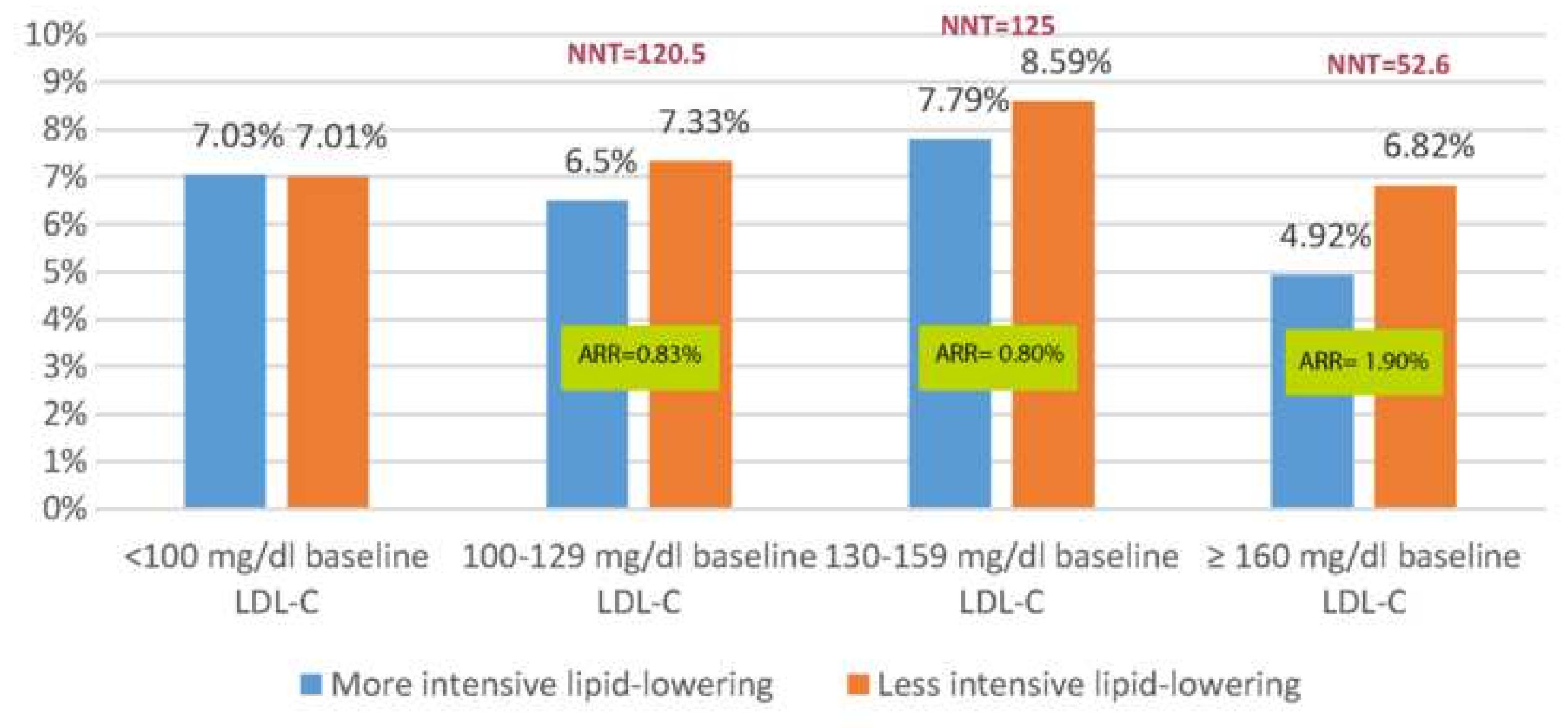

NNT $=125$
LDL-C
LDL-C
LDL-C
More intensive lipid-lowering $\quad$ Less intensive lipid-lowering

LDL-C 


\section{MACES}

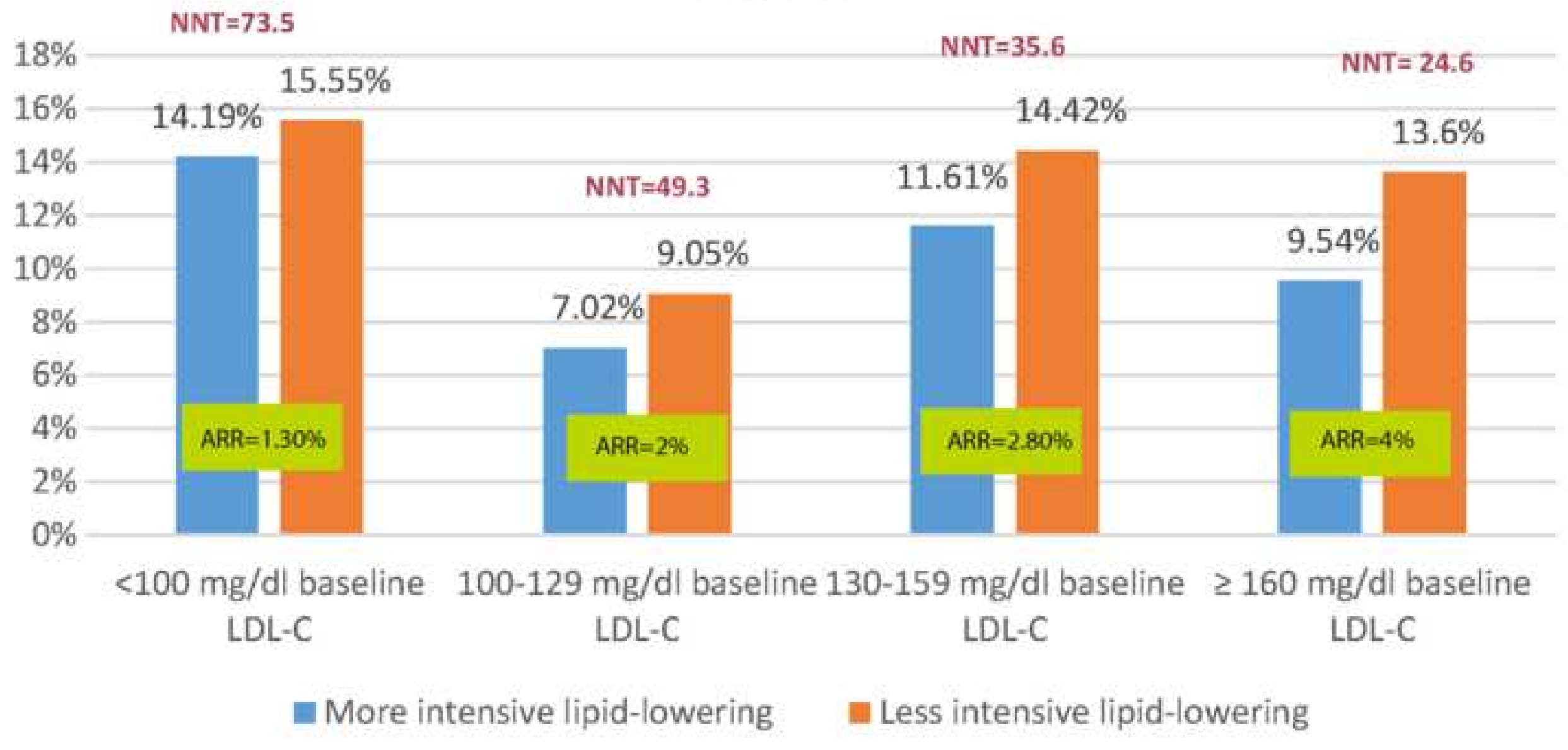




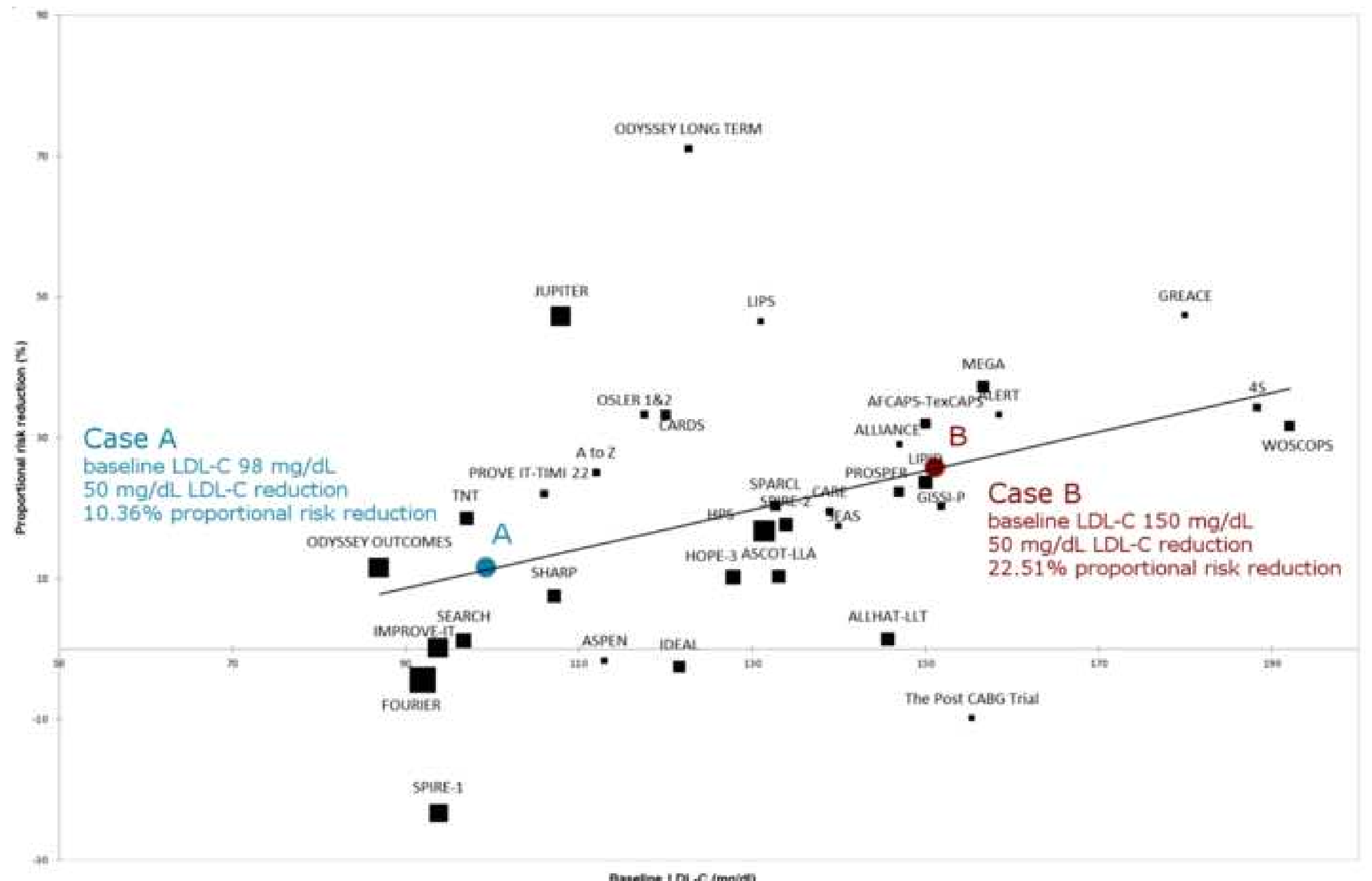

baseline LDL-C $98 \mathrm{mg} / \mathrm{dL}$.

$50 \mathrm{mg} / \mathrm{dL}$ LDL.C reduction

oOYSSEY OUTCOMES

SPIIRE-1
MLLAT-LT

. 


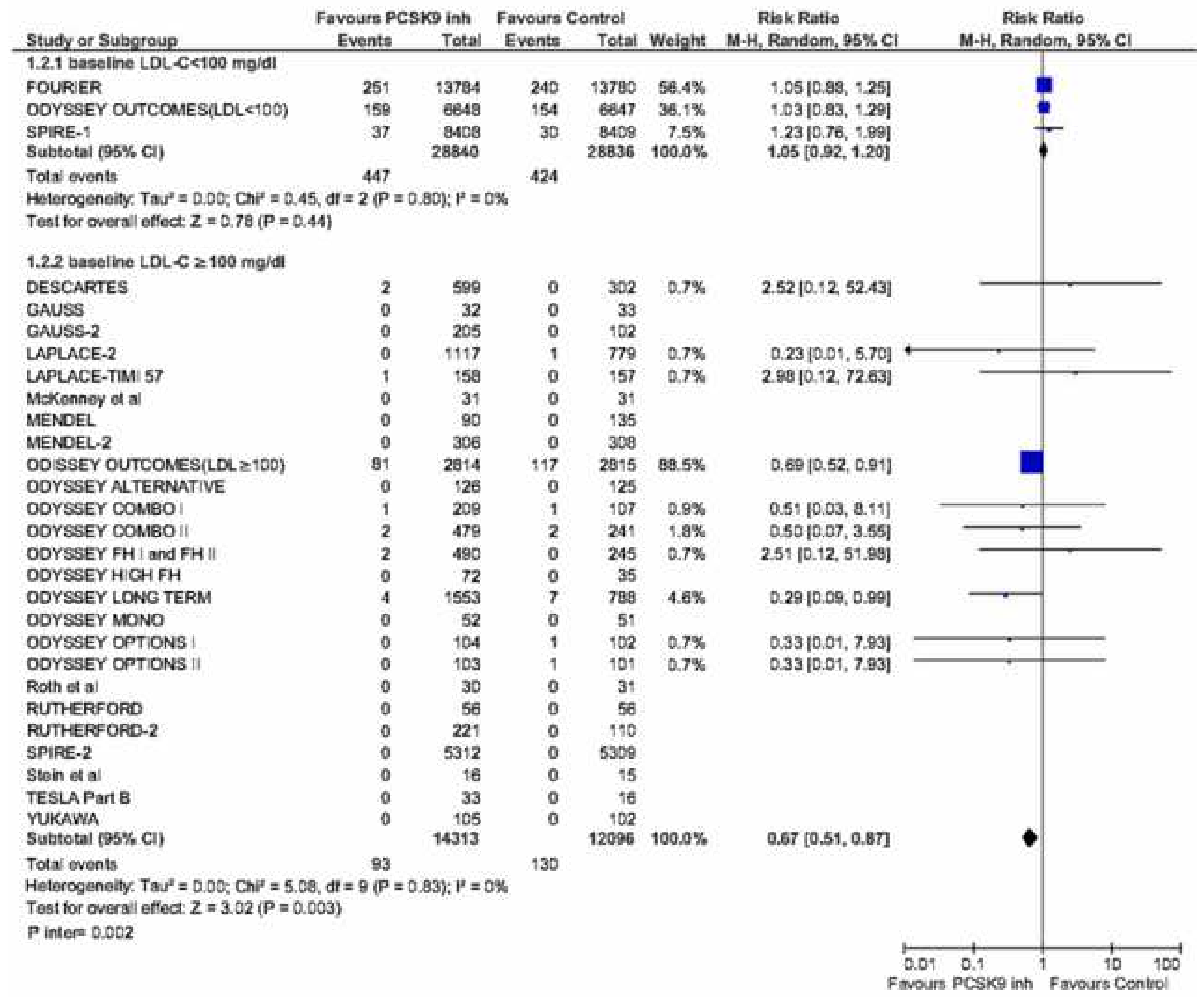

Total Events Total Weight M.H. Random, 
Click here to access/download

\section{Supplementary files suppl figures (1).docx}


Dr Navarese reported receiving research grants from Amgen and personal fees from Amgen and Sanofi.

Andreotti reported receiving personal fees from Actelion, Amgen, Bayer, Boehringer-Ingelheim; Bristol-Myers Squibb, Pfizer, Daiichi Sankyo, and the Menarini International Foundation.

Dr Tantry reported receiving personal fees from AstraZeneca and Medicure.

Dr Raggi reported receiving grants from Amgen and Sanofi.

Dr Gurbel reported receiving a grant from Amgen. 